\title{
MIAWARE SOFTWARE \\ 3D Medical Image Analysis With Automated Reporting Engine and Ontology-based Search
}

\author{
Bartłomiej Wilkowski \\ Informatics and Mathematical Modelling, Technical University of Denmark, Lyngby, Denmark \\ bw@imm.dtu.dk
}

Óscar N. M. Pereira, Paulo Dias

IEETA-Institute of Electronics and Telematics Engineering of Aveiro, University of Aveiro, Aveiro, Portugal

omp@ua.pt,paulo.dias@det.ua.pt

Miguel Castro

Centro Hospitalar do Baixo Alentejo - Hospital José Joaquim Fernandes de Beja, Beja, Portugal

migkastro@yahoo.com

Marcin Janicki

Department of Microelectronics and Computer Science, Technical University of Łódź, al. Politechniki 11, Poland

janicki@dmcs.pl

Keywords: Computed axial tomography; Ontology; Radiological report; Image visualization;

\begin{abstract}
This article presents MIAWARE, a software for Medical Image Analysis With Automated Reporting Engine, which was designed and developed for doctor/radiologist assistance. It allows to analyze an image stack from computed axial tomography scan of lungs (thorax) and, at the same time, to mark all pathologies on images and report their characteristics. The reporting process is normalized - radiologists cannot describe pathological changes with their own words, but can only use some terms from a specific vocabulary set provided by the software. Consequently, a normalized radiological report is automatically generated. Furthermore, MIAWARE software is accompanied with an intelligent search engine for medical reports, based on the relations between parts of the lungs. A logical structure of the lungs is introduced to the search algorithm through the specially developed ontology. As a result, a deductive report search was obtained, which may be helpful for doctors while diagnosing patients' cases. Finally, the MIAWARE software can be considered also as a teaching tool for future radiologists and physicians.
\end{abstract}

\section{INTRODUCTION}

This article presents MIAWARE, a software, which enables doctors and radiologists to carry out an examination of the patient's lungs condition through the close analysis of the computed axial tomography images and then, in parallel, to perform health state reporting process. Secondly, an intelligent search engine for radiological reports is presented, together with all its advantages over the ordinary searching schemas. The screenshot of the MIAWARE's application graphical user interface is shown in Figure 1. MIAWARE was developed completely in Java programming language together with some embedded native code wrappers used.
Nowadays, it is very common that a radiologist performs the analysis of the radiological images in its own, favorite manner. Some of the radiologists report all pathological changes encountered in the radiological images speaking to a microphone and recording their voice. Afterwards, the recorded tape is listened out and a medical text report is produced. Another radiologists write reports alone in the moment of performing analysis.

There can be found some serious shortcomings in such reporting schemas, which may affect the accuracy of the medical diagnoses. The main problem is that reports differ in structure from radiologist to radiologist. Every human has different way of thinking, different way of expressing things, remarks and 


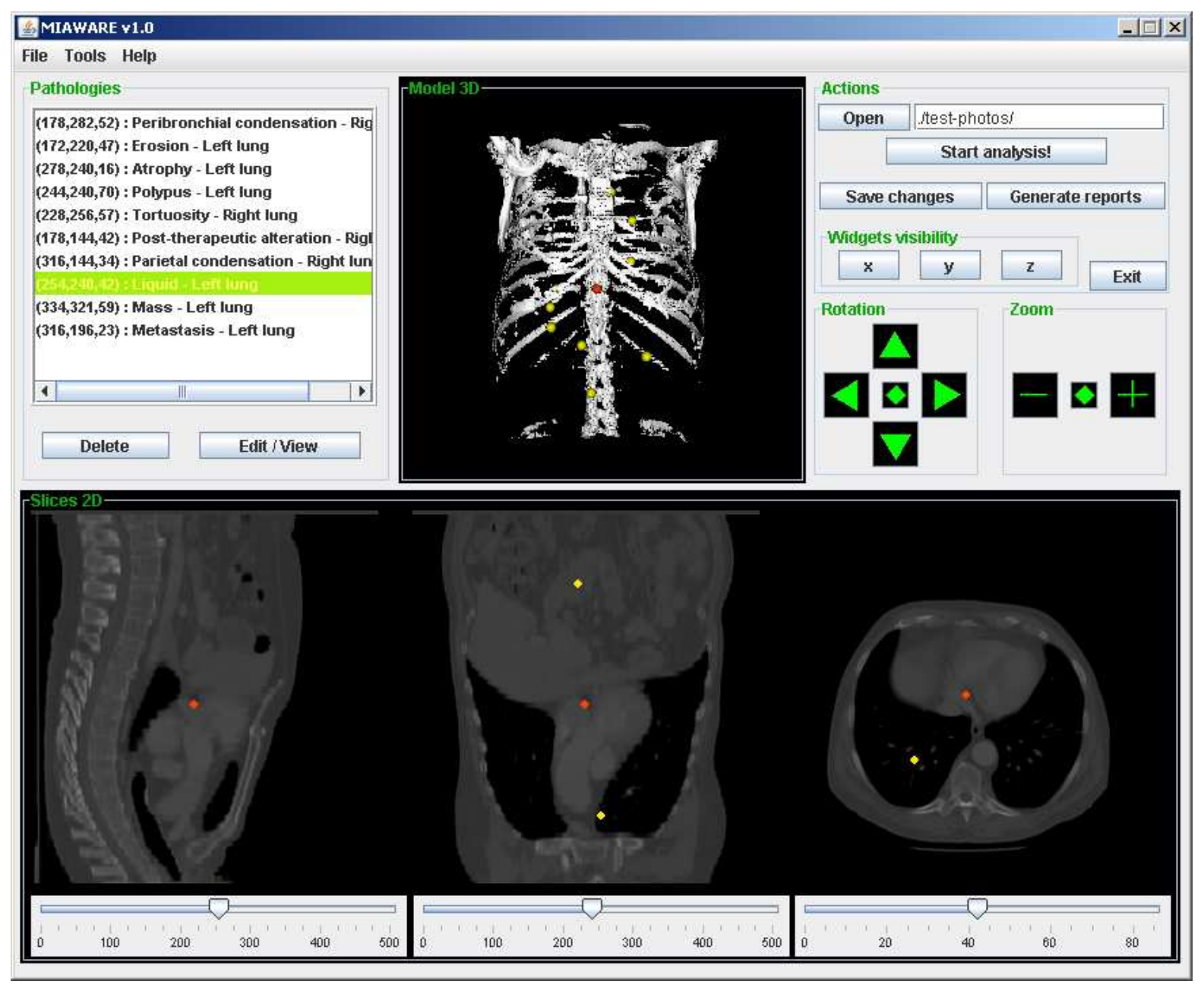

Figure 1: The graphical user interface of the MIAWARE software. The radiologist is able to cut the 3D model in order to find pathologies on the 2D views, mark them by simple mouse click and attach necessary description. All pathologies are listed out in the Pathologies panel. After the examination, the normalized medical report can be generated.

observations. It means that given the same medical data, the same patient's case to many radiologists in order to make analysis, it can and will, almost surely, produce many different reports with different layouts and various observations on the patient's health. As a result, a doctor may interpret each of such reports differently.

This is the reason why MIAWARE software's main objective is to generate medical reports in a normalized way. Such reports should contain only medical data, which describes in details the encountered pathologies using a standardized layout. The radiologist does not use his words in order to report a pathology, but oppositely, he fills up a provided reporting form by choosing suitable medical terms.

Moreover, pathology reporting in MIAWARE is performed in the moment of image analysis using interactive graphical user interface. Radiologist can mark the location of the pathology on the image and associate with this point a necessary description. This allows him to be always focused on the images. Finally, a normalized medical report over all pathological changes is generated by the software. The full description of how a normalized reporting process is performed with MIAWARE software is described in details in section 3 .

Normalization of the reports improves significantly its further processing possibilities. One example can be the developed search engine for the MIAWARE medical reports. An efficient search engine for medical reports can be considered as very useful and may help the doctor while making diagnoses.

Further sections will describe in details the architecture of the MIAWARE software and the functionality of its modules. 


\section{VISUALIZATION OF IMAGES}

The entire visualization of CAT scan stack images and 3D model creation is performed using the $\mathrm{Vi}$ sual Toolkit (VTK - www.vtk.org) (Schroeder et al., 1998). VTK is made in C++ language, but it provides suitable wrapper classes for Java. Moreover, ImageJ (http://rsb.info.nih.gov/ij/) software classes are used in order to obtain properties of the analyzed CAT image stack.

MIAWARE graphical user interface provides a 3D view of the radiological stack of images. This is generated using VTK wrapper classes, which create a special pipeline. After loading image data into memory, a contour filter is applied followed by proper mapping of polygonal data and graphics primitives. Finally, a 3D actor is added to the special panel, which actually is a rendering window for three-dimensional scene.

The visualization in MIAWARE consists also of three 2D cross-sectional image views. They are generated by three widgets (interactive window objects), present on the 3D scene, which are able to cut the model in three plane directions and provide 2D image data for the cross-sectional views (see Figure 2). Widgets can be easily moved by the radiologist along its respective direction axis in order to perform model cutting. It should be mentioned, that CAT scan provides the radiologist with image stack in axial plane. The image data for two other $2 \mathrm{D}$ planes and the 3D model are obtained and rendered by the software after a proper initial stack data processing.

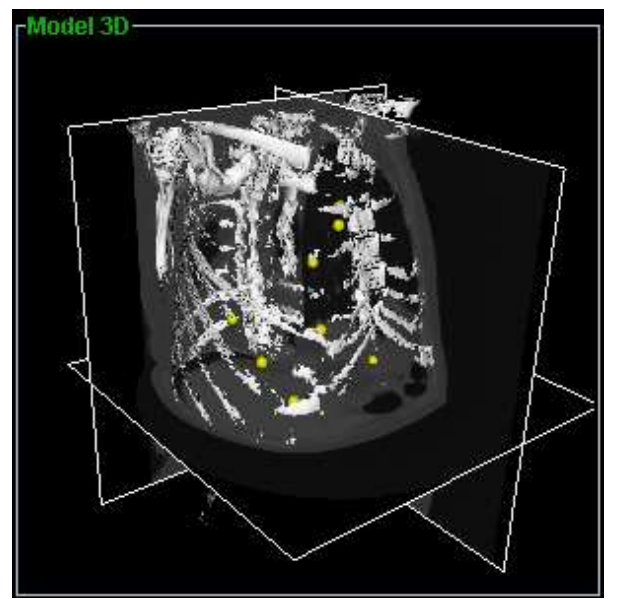

Figure 2: A view of the 3D model with three visible cutting widgets.

Finally, the panels, which display cross-sectional views of the model are enhanced with a very important feature. Radiologist is able to mark any pathology, encountered during the analysis, directly on the $2 \mathrm{D}$ view by a simple left mouse button click over that location. The clicked point is automatically marked on all three cross-sectional views (as a yellow circle) and 3D scene (yellow sphere). Afterwards, the radiologist is able to attach precise information and description of that physiological change to the marked point. The description of how the pathology information is defined and added to the specified location is presented in section 3. It should be also remembered that all the pathologies defined by the radiologist can be saved and retrieved for further analysis of the same CAT stack.

\section{PATHOLOGY REPORTING}

As it was mentioned in the introductory part of this article, the reports generated with MIAWARE software are normalized. This is achieved thanks to a special pathology reporting form implemented in this software. According to the previous section, radiologist is able to mark any location on the $2 \mathrm{D}$ image in order to define and describe the encountered pathology. Such an information is added through a comboboxbased form, which provides radiologist with medical terms necessary for an effective name, type and pathology location specification. The most innovative here is the fact that the radiologist cannot describe those findings with his own words, but can use only the specific medical vocabulary provided by the application. Consequently, MIAWARE software is able to create normalized medical reports according to the information about all pathologies introduced earlier by the radiologist.

Arrangement and selection of the vocabulary was made after the consultation with doctor Miguel Castro working in hospital in Beja (Centro Hospitalar do Baixo Alentejo - Hospital José Joaquim Fernandes de Beja) and RadLex (A Lexicon for Uniform Indexing and Retrieval of Radiology Information Resources) term browser, which can be found on the Radiological Society of North America web page (RSNA.org, 2007). RadLex term browser was created in order to unify the radiological vocabulary used during image analysis and reporting procedures.

The entire vocabulary is kept in the XML file together with a declaration of the vocabulary for all comboboxes (set of medical terms), which are presented to the radiologist during the pathology definition. A vocabulary set presented in any subsequent combobox is dependent of a previous radiologist's choice. For example, if radiologist has defined that the pathology is located in the left lung, the next combobox will offer him to choose all subparts (lobes) of left lung. The example pathology definition steps in MIAWARE is presented in Table 1. 


\begin{tabular}{|c||c||c|}
\hline Step & Combobox title & Selected value \\
\hline \hline 1. & Morphophysiological process & Neoplastic process \\
\hline 2. & Neoplastic process & Mass \\
\hline 3. & Location & Left lung \\
\hline 4. & Left lung location & Upper lobe \\
\hline 5. & Left lung upper lobe location & Lingula \\
\hline 6. & Left lung upper lobe lingula location & Superior segment \\
\hline
\end{tabular}

Table 1: Example pathology definition steps in MIAWARE.

When the analysis of the CAT stack is finished, radiologist is able to generate a final medical report over all the pathologies already defined. It is done by pressing the Generate reports button. This action produces reports in two formats: Plain Text Format (TXT) and Rich Document Format (RDF www.w3c.org/RDF/), computer understandable format. The first one can be verified and analyzed later by the doctor in order to make diagnosis. The generated text report has a well defined structure and its layout differs significantly from the recently created reports. The format of medical reports requires still some discussion over its layout and the ways how it should be created. MIAWARE text report format is only a suggestion, which is intended for further improvement and development. A short fragment of the sample MIAWARE text report is presented here:

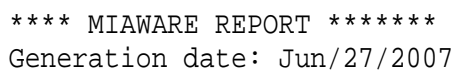

The second type of reports, in RDF format, is created for further processing of its content (report searching). It is described in details in section 4 .

\section{MEDICAL REPORT SEARCHING}

This section describes the structure of the RDF medical reports and the MIAWARE search engine together with an ontology for lungs developed specially for this purpose.

\subsection{RDF reports}

As it was already mentioned, the RDF format for medical reports is required for further information processing and searching. RDF model introduces description of resources by statements and its data model contains of three components: resources, properties and statements (called as triples). Resources are any datatype items, which can have any value definition (statement) through some given relation (property). Any statement can consist of a new triple resource-property-statement. "Just as an English sentence usually comprises a subject, a verb and objects, RDF statements consist of subjects, properties and objects" (Gomez-Perez et al., 2004).

Table 1 represents one example of pathology definition. The final medical report will usually contain more such definitions grouped in some specific way. The data gathered in the Table 1 can be represented as normal, lexical group of sentences describing any pathology found. For example:

'A morphophysiological process was found. It is in the form of a neoplastic process of the type mass. It is located in the left lung, in its upper lobe, exactly in the superior segment of the lingula.'

Such a group of sentences can be represented as resource-property-statement model and is used in MIAWARE medical RDF reports. In this case, the first underlined word is a resource and the rest is a statement. As our statement consists of group of resources it has to be analyzed further. Then the first resource of the previous statement is a resource and the rest group another statement. Such embedded structure of the resource-property-statement is created 
through RDF reified statements. It should be mentioned that the properties (which connect resources with the statements) in the above example are: in form of, of the type, etc.

The RDF reports generated by MIAWARE software keep the pathology information in the manner presented above. It should be only mentioned that the role of properties in our reports play titles of the subsequent comboboxes. These names are taken from the XML file used by pathology definition form, described in section 3 .

\subsection{Ontology-based report searching}

The radiological examinations are carried out quite often in such places as hospitals, private and public surgeries or any other medical institutions. As a result, it produces a great amount of medical reports in relatively short time. Such reports should be kept and gathered together for future usage as references to previously encountered and defined pathologies or diseases. Manual searching of great amount of documents is time consuming. As a result, an intelligent search engine of medical reports can significantly speed-up the disease recognition process, as, considering given criteria, it would immediately result in sets of references to the archive reports with similar pathological symptoms in other patients, the resultant diagnoses and applied treatments.

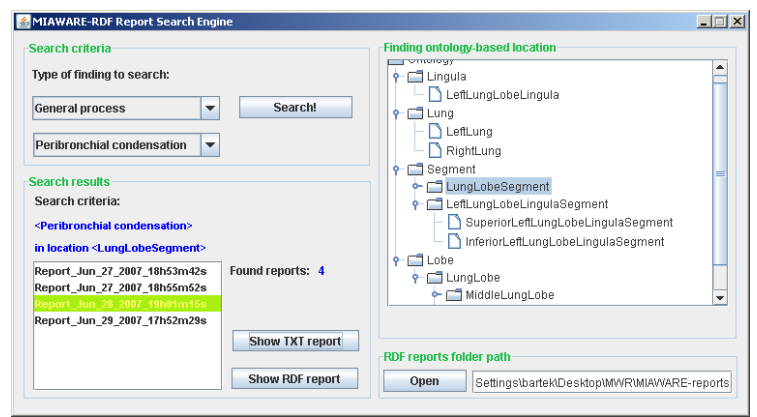

Figure 3: The graphical user interface of the MIAWARE intelligent search engine. The sets of RDF medical reports generated by MIAWARE software can be filtered out according to the given criteria: position in lungs and pathology type. The searching process is performed on a logical basis (not in a lexical way, as ordinary search engines).

The search engine for medical reports developed together with MIAWARE software (Figure 3) is able to find all reports where exist some specified pathology defined in a lung part (specified as a search criteria) or any of its subparts. This adds some intelligence to the searching process, what is explained on the simple example. Let's suppose that a doctor wants to find all reports with a definition of a tumour (first search criterion), which had been found in left lung. Let's have a report with two pathologies defined:

- Polypus in Right lung

- Tumour in Lung lingula

An ordinary lexical search will respond that this report does not match search criteria as the first pathology is not a tumour and the second pathology, which is a tumour, is not located in left lung. Oppositely, the MIAWARE search engine will accept this report as matching the given criteria, because it can deduce that Lung lingula is a subpart of the left lung. Such a logical deduction is performed by our search engine thanks to the lungs ontology, which defines and provides the part-whole relations between the elements of the lungs. This ontology was developed using Jena (http://jena.sourceforge.net/) and Protégé (http://protege.stanford.edu/) software. Sample visualization of the taxonomy of the classes taken from our lungs ontology is presented in Figure 4.

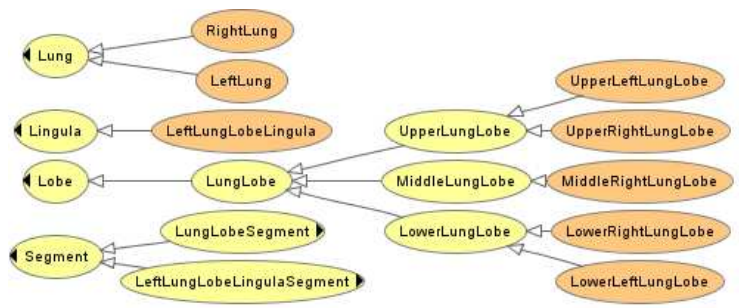

Figure 4: Lungs ontology - hierarchy of classes (created with OWLViz (Drummond, 2007)). Such a taxonomy of classes, related to each other through the specific properties defined in an ontology, allow the search engine to make intelligent (logical) decisions.

Our ontology was developed based on the following article references: (Mejino Jr et al., 2003) (Donnelly et al., 2005) (Guarino and Welty, 2000) (Guarino and Welty, 2004) (Guarino et al., 2000) (Guarino and Welty, 2002) (Michael et al., 2001) (Knublauch, 2004) and book positions: (Gomez-Perez et al., 2004) (Horridge, 2004). Moreover, the information about anatomical structure of the lungs was taken from Anatomy and Physiology book (Seeley et al., 2005). The ontology is made in Web Ontology Webpage OWL (www.w3c.org/2004/OWL/) format.

The search algorithm takes as the criteria the name of the pathology and its location in the lungs. Next, it deduces from the ontology all the subparts of the given lung location and performs comparison of every single pathology description taken from any medical report (in RDF format) with the search criteria. If there is at least one such a pathology definition which agrees with criteria, it displays respective report as a result. Consequently, the doctor can view and read 
such a report very easily. We suppose that such filtering of radiological reports may improve doctor's diagnosis and speed-up his decisions.

\section{CONCLUSIONS}

The presented software is only a first prototype and needs many improvements to be useful in a real context. One of the reason for this is the fact that the vocabulary used during pathology reporting is not sufficient and requires significant expansion and redefinition. However, this software can be considered as a strong fundament for future development in order to achieve a fully operational version.

The ideas presented herein are considered as a potential improvement for image-based medicine and radiological analysis course. MIAWARE software facilitates radiologists with simultaneous analysis of the CAT stack images and pathology reporting without looking away from the monitor. Consequently, the radiologist can be concentrated all the time on the examined images. Moreover, pathologies can be marked on the images and possess the necessary characteristics of respective pathology.

Furthermore, the radiological reports generated with MIAWARE software are always normalized, keeping identical structure and layout independently on the person who performs the analysis. Such a normalization, may help the doctors in better understanding of the reports and it makes room for further report processing and searching.

The intelligent search engine allows rapid medical reports filtering according to the pathologies defined in there. Providing MIAWARE search engine with the knowledge about the parts relationship in the lungs, it is able to deduce internal elements of the specified lung part and to perform report searching of the pathologies not only in the determined lung location, but also in its subparts. This can actually be described as a logical searching of pathologies in the medical reports.

All the features presented by MIAWARE software can lead to the assumption that their implementation into real life may result in more efficient medical diagnosis and faster disease recognition process. Moreover, MIAWARE can be used for investigation and teaching of normalized reporting processes, pathologies and findings classification, statistical processing, etc. Thanks to that, the future radiologist could get their degree through intensive practice with real cases. Finally, the reports generated by the students using MIAWARE software could be evaluated in an automatic manner.

\section{ACKNOWLEDGEMENTS}

This work is supported by Lundbeckfonden through the program www.cimbi.org.

\section{REFERENCES}

Donnelly, M., Bittner, T., and Rosse, C. (2005). A formal theory for spatial representation and reasoning in biomedical ontologies. Artificial Intelligence in Medicine, 36:1-27.

Drummond, N. (2007). Owlviz - ontology visualization tool webpage.

Gomez-Perez, A., Corcho, O., and Fernandez-Lopez, M. (2004). Ontological Engineering : with examples from the areas of Knowledge Management, e-Commerce and the Semantic Web. First Edition (Advanced Information and Knowledge Processing). Springer.

Guarino, N. and Welty, C. A. (2000). A formal ontology of properties. Lecture Notes in Computer Science, 1937:97-112.

Guarino, N. and Welty, C. A. (2002). Evaluating ontological decisions with ONTOCLEAN. Communications of the ACM, 45:61-65.

Guarino, N. and Welty, C. A. (2004). An overview of ontoclean. Handbook on ontologies, pages 151-172.

Guarino, N., Welty, C. A., and Partridge, C. (2000). Towards a methodology for ontology based model engineering. IWME-2000 - International Workshop on Model Engineering.

Horridge, M. (2004). A Practical Guide To Building OWL Ontologies With The Protege-OWL Plugin. University of Manchester, 1 edition.

Knublauch, O. H. (2004). Weaving the biomedical semantic web with the protege owl plugin.

Mejino Jr, J. L., Agoncillo, A. V., Rickard, K. L., and Rosse, C. (2003). Representing complexity in partwhole relationships within the foundational model of anatomy. American Medical Informatics Association Annual Symposium, pages 450-454.

Michael, J., Mejino Jr, J. L., and Rosse, C. (2001). The role of definitions in biomedical concept representation. American Medical Informatics Association Fall Symposium, pages 463-467.

RSNA.org (2007). Rsna - radlex term browser webpage.

Schroeder, W., Martin, K., and Lorensen, B. (1998). The Visualization Toolkit- An Object Oriented Approach to $3 D$ Graphics. 2nd ed. Prentice Hall.

Seeley, R. R., Stephens, T. D., and Tate, P. (2005). Anatomy and Physiology. McGraw-Hill Higher Education. 